\title{
Massenhaushalt des Laika Glacier, Coburg Island, kanadischer arktischer Archipel
}

Coburg Island $\left(75^{\circ} 54^{\prime} \mathrm{N}, 79^{\circ} \mathrm{W}\right)$ hat eine Länge von etwa $35 \mathrm{~km}$ und eine mittlere Breite von $10 \mathrm{~km}$ und liegt an der SE-Ecke der Ellesmere Island, an der Mündung des Jones Sound in die Baffin Bay. Der höchste Punkt liegt $820 \mathrm{~m}$ ü.M. Der südöstliche Teil weist flachere Regionen auf. Die Insel ist stark vergletschert, mit einem Maximum an der NW-Küste. Dort erreichen die meisten Gletscher das Meer und bilden Eisberge. Das Laika Ice Cap liegt auf einer nach SE gerichteten Halbinsel auf einem runden, an seiner Kulmination $520 \mathrm{~m}$ hohen Hochplateau, das leicht nach NE geneigt ist.

Das Klima der Insel wird durch die North Water Polynya stark beeinflußt. Die Durchschnittstemperaturen im Winter (Januar bis März) liegen höher (6 bis $8^{\circ} \mathrm{C}$ ) als bei den benachbarten arktischen Stationen, die nicht durch das offene Wasser der Polynya beeinflußt sind. Auch die Niederschläge sind durch die Polynya um mindestens einen Drittel erhöht, wobei

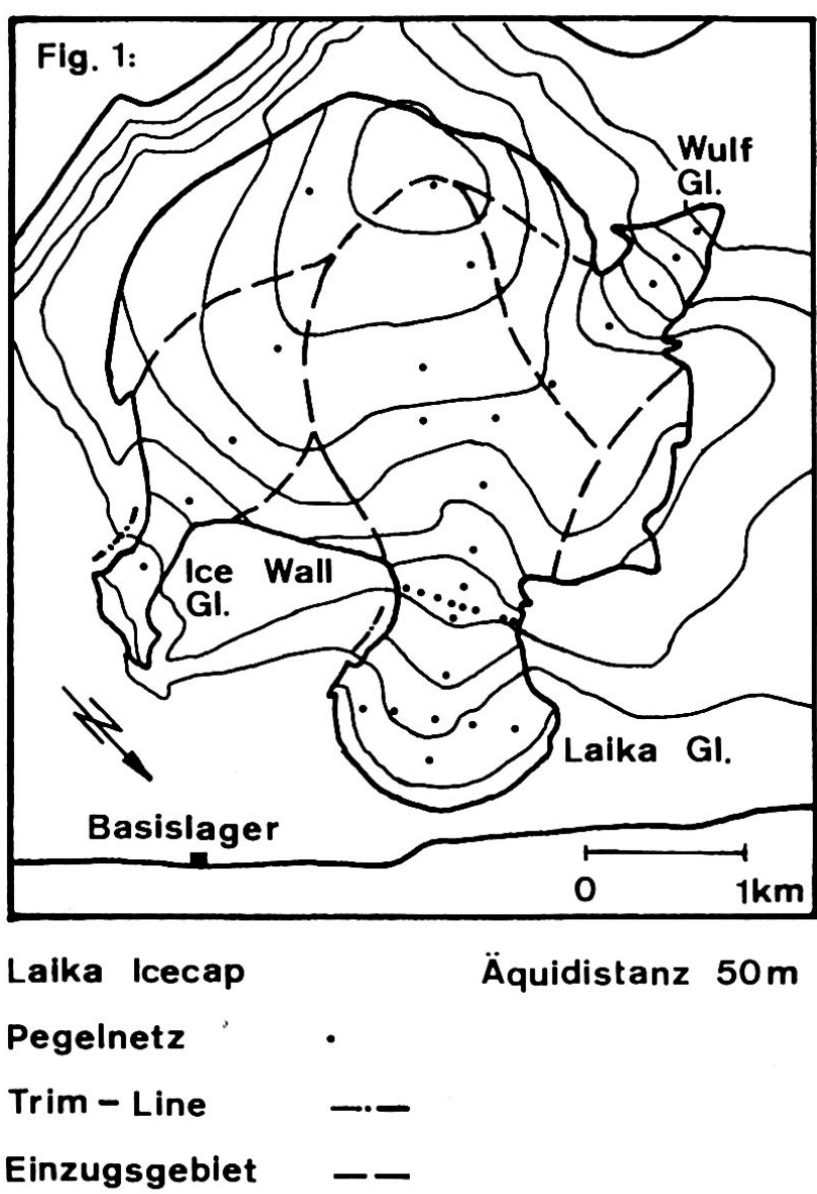

das Maximum im Juli und August auftritt. Da die Insel recht exponiert liegt, wird ihr Klima stark durch die Winde geprägt. Die dominanten Winde blasen von $\mathrm{W}$ und $\mathrm{NW}$.

Das Laika Ice Cap (Fig. 1) ist eine kreisförmige Eismasse von knapp $10 \mathrm{~km}^{2}$ Fläche. Der größte Teil des Eises fließt in nördliche Richtung ab. Die Eiskappe besitzt drei Ausflußgletscher. Der Wulf Glacier mit nur $1,3 \mathrm{~km}^{2}$ Fläche fließt steil nach Westen. Er ist im Rückzug begriffen und erreicht die westliche Talebene heute nicht mehr. Im $\mathrm{E}$ befindet sich der Ice Wall Glacier, der mit einer Fläche von $1,7 \mathrm{~km}^{2}$ in einer Steilwand endet. Er wird vorwiegend durch Driftschnee ernährt. Auf der windabgewandten Talfläche wird durch den Windschatteneffekt eine riesige Schneewächte aufgebaut, die den eigentlichen Oberbau des Ice Wall Glacier bildet. Der Laika Glacier beansprucht mit seinen $4,3 \mathrm{~km}^{2}$ nahezu die Hälfte der Eiskappe und erstreckt sich mit gleichmäßigem Gefälle vom höchsten Punkt bis beinahe auf das Meeresniveau. Das Längsprofil weist nur zwei kleinere Steilstufen auf. Nach dem Durchfluß durch die engste Stelle weitet sich die Zunge piedmontartig auf der davor liegenden Strandebene aus. Auf der östlichen Zungenseite befindet sich eine das Eis bedeckende Moräne, die auf der westlichen Seite klar von Gletschereis getrennt ist.

\section{Massenbilanz}

Es wurde die direkte glaziologische Methode mit natürlichem Haushaltsjahr verwendet. Die Nettobilanz $B_{n}$ ergibt sich aus der Summe von Akkumulation und Ablation auf dem ganzen Gletscher; $\mathrm{B}_{\mathrm{n}}$ wird in $\mathrm{m}^{3}$ Wasseräquivalent (w.e.) angegeben (HOINKEs, 1970). Es gilt also, auf dem Gletscher die wichtigen Zeitpunkte der maximalen und der minimalen Masse zu erkennen. Die Nettobilanz wird durch ein Netz von 34 Stangen auf dem Gletscher erfaßt. Gemessen werden:

a) die Schnee- und Eisrücklagen, die am Ende des Haushaltsjahres im Akkumulationsgebiet verbleiben, 
b) die Schnee- und Eismassen, die während des Haushaltjahres im Ablationsgebiet verloren gehen.

Bei den Messungen auf dem Laika Glacier (bzw. Ice Cap) wurde versucht, die (maximale) Winter- und die (minimale) Sommermasse zu erfassen. Die gemessenen Werte werden auf einer topographischen Karte eingetragen und Isolinien gleichen Gewinnes oder Verlustes gezeichnet. Durch Planimetrierung der Flächen zwischen den Isolinien kann das gewonnene, respektive verlorene Wasservolumen bestimmt werden. Es standen im Rahmen des North Water Projektes (McGill Universität und ETHZ) Massenhaushaltsdaten für die Jahre 1973/74, 1974/75 und 1975/76 zur Verfügung.

\section{Ergebnisse}

Die Daten des erstgenannten Jahres wurden von KAPPENBERGER (1974) zusammengestellt. Die Schneeablagerungen bleiben infolge der erwähnten Winde selten für längere Zeit ungestört liegen, woraus eine recht charakteristische, inhomogene Schneeverteilung resultiert. Auf dem Laika Ice Cap spielt der Massengewinn durch aufgefrorenes Eis (superimposed ice) eine große Rolle. Im Gegensatz zur gewöhnlich langsam erfolgenden Umwandlung von Schnee zu Eis gefriert dabei Schmelzwasser, das durch die Schneedecke auf die noch kalte Gletscheroberfläche sickert. Gletscher, welche sich zu einem großen Teil durch

FIG. 2:
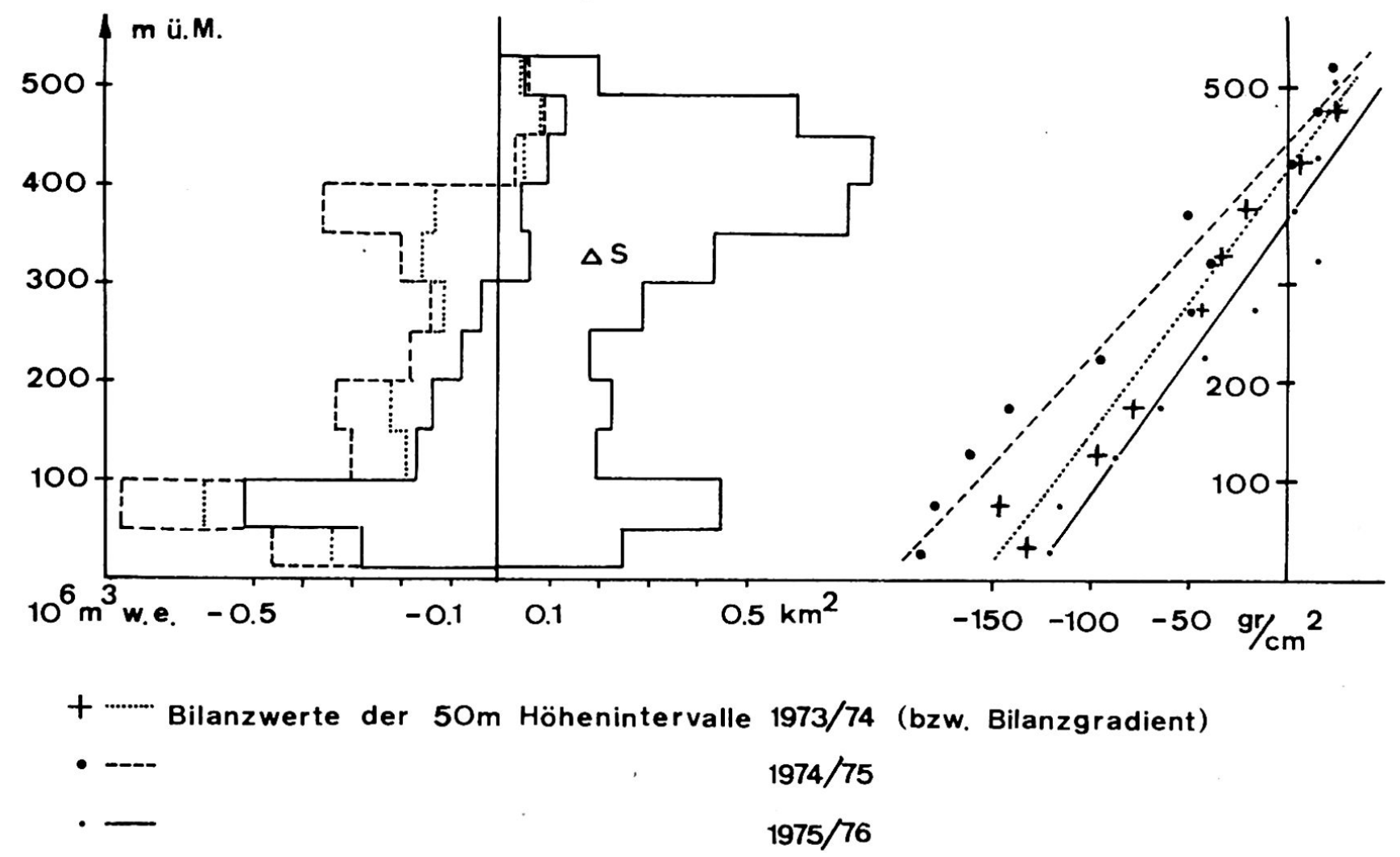

$\Delta S$ Flächenwerte der $50 \mathrm{~m}$ Höhenintervalle des Laika Glacier 
aufgefrorenes Eis erhalten, werden zum Baffin-Typ (BAIRD, 1952) gezählt. Dieser Prozeß kann auf dem Laika Ice Cap während des ganzen Sommers aktiv sein. Die Schmelze beginnt in der Regel im ersten Drittel des Monats Juni. Gebiete mit - infolge dünner Winterschneedecke - früh einsetzender Eisschmelze weisen am Ende des Haushaltsjahres den höchsten Wasserverlust auf.

Die Massenbilanz des Laika Glacier ist für alle drei Beobachtungsjahre (Fig. 2, Tab. 1) negativ, wobei unterschiedliche Werte von Jahr zu Jahr gemessen werden. Der Verlust des Haushaltjahres 1975/76 ist rund 3,5mal kleiner als jener des Vorjahres. Die Massenbilanzresultate spiegeln sich in der variierenden Größe der Akkumulationsgebiete wider. Das Verhältnis von Akkumulationsgebiet zur gesamten Gletscherfläche wird durch den sogenannten AAR (accumulation aero ratio)-Wert (Tab. 2) angegeben. Für 1975/76 ist der Wert 0,5, während er für das Vorjahr nur 0,3 betrug. Daraus ergibt sich, daß für einen ausgeglichenen Massenhaushalt der AAR-Wert des Laika Glacier größer als 0,5 , schätzungsweise 0,6 bis 0,7 sein muß. Wie allgemein gültig bedeutet auch im Falle des Laika Glacier eine Verkleinerung des AAR-Wertes einen Anstieg der Gleichgewichtslinie. Die Höhe dieser Trennungslinie (Tab. 4) zwischen Akkumulations- und Ablationsgebiet hängt mit der Temperaturverteilung während der Ablationszeit zusammen.

Die Summe der positiven Sommertemperaturen ist ein $\mathrm{Maß}$ für die Temperaturverhältnisse. Von den drei beobachteten Jahren weist das Haushaltsjahr 1975/76 mit dem kleinsten Wasserverlust ebenfalls die kleinste positive Temperatursumme auf (Tab.3). Die Abhängigkeit der Massenbilanz von den Niederschlägen ist weniger eindeutig. Das Jahr mit dem größten Massenverlust (1974/75) weist zugleich den höchsten Sommerniederschlag (Mai-August) auf. Entscheidend ist wahrscheinlich nicht die Niederschlagssumme, sondern deren Verteilung. Während der Sommer 1974 bzw. 1975 fielen in den Monaten Juli und August nur an einem, bzw. keinem Tag Schnee beim Basislager, wobei nur Tage mit einem meßbaren Niederschlag von mindestens $0,025 \mathrm{~cm}$ w.e. berücksichtigt wurden.

Tab. 2: AAR-Werte

$\begin{array}{ll}\text { a) Laika Glacier: } & 73 / 74: 0,27 \\ & 74 / 75: 0,27 \\ & 75 / 76: 0,5 \\ \text { b) Icecap: } & 74 / 75: 0,15 \\ & 75 / 76: 0,33\end{array}$

Tab. 3: Summe der pos. Sommertemperatur, gemessen am Basislager

1974: $277\left({ }^{\circ} \mathrm{C}\right)$

1975: $312\left({ }^{\circ} \mathrm{C}\right)$

1976: $202\left({ }^{\circ} \mathrm{C}\right)$

Tab. 4:

\begin{tabular}{lccc}
\hline & $73 / 74$ & $74 / 75$ & $75 / 76$ \\
$\begin{array}{lcc}\text { Bilanzgradient } \\
\text { cm w.e. / } 100 \mathrm{~m}\end{array}$ & 36 & 48 & 35 \\
$\begin{array}{l}\text { Höhe der Gleichgewichts- } \\
\text { linie m ü. M. }\end{array}$ & 420 & 455 & 360 \\
\hline
\end{tabular}

Tab. 1: Bilanzen des Laika Icecap.

\begin{tabular}{|c|c|c|c|c|c|c|c|c|c|c|c|}
\hline \multirow[b]{2}{*}{$\begin{array}{l}\text { Fläche S } \\
\text { Jahr }\end{array}$} & \multirow{2}{*}{$\begin{array}{l}\text { Benutzte } \\
\text { Einheit } \\
\mathrm{km}^{2}\end{array}$} & \multicolumn{3}{|c|}{ Laika Gl. } & \multicolumn{2}{|c|}{ Wulf Gl. } & \multicolumn{2}{|c|}{ Ice Wall Gl. } & \multicolumn{2}{|c|}{ Restflächen } & Laika Icecap \\
\hline & & $\begin{array}{l}4,27 \\
73 / 74\end{array}$ & $74 / 75$ & $75 / 76$ & $\begin{array}{l}1,29 \\
74 / 75\end{array}$ & $75 / 76$ & $\begin{array}{l}1,69 \\
74 / 75\end{array}$ & $75 / 76$ & $\begin{array}{l}2,58 \\
74 / 75\end{array}$ & $75 / 76$ & $\begin{array}{ll}9,83 & \\
74 / 75 \quad 75 / 76\end{array}$ \\
\hline $\begin{array}{l}\text { Winterbilanz } B_{W} \\
b_{w}=B_{W} / S\end{array}$ & $\begin{array}{l}10^{6} \mathrm{~m}^{3} \text { w.e. } \\
\mathrm{cm} \text { w.e. }\end{array}$ & $\begin{array}{l}+1,69 \\
+39,6\end{array}$ & $\begin{array}{l}+1,95 \\
+45,7\end{array}$ & $\begin{array}{l}+0,77 \\
+18\end{array}$ & $\begin{array}{l}+0,52 \\
+40,3\end{array}$ & $\begin{array}{l}+0,16 \\
+12,4\end{array}$ & $\begin{array}{l}+0,81 \\
+47,9\end{array}$ & $\begin{array}{l}+0,5 \\
+29,1\end{array}$ & $\begin{array}{l}+0,97 \\
+37,6\end{array}$ & $\begin{array}{l}+0,45 \\
+17,4\end{array}$ & $\begin{array}{l}+4,25+1,88 \\
+43,2+19,1\end{array}$ \\
\hline $\begin{array}{l}\text { Sommerbilanz } B_{S} \\
b_{S}=B_{S} / S\end{array}$ & $\begin{array}{l}10^{6} \mathrm{~m}^{3} \text { w.e. } \\
\mathrm{cm} \text { w.e. }\end{array}$ & $\begin{array}{l}-3,4 \\
-79,6\end{array}$ & $\begin{array}{l}-4,3 \\
-100,7\end{array}$ & $\begin{array}{l}-1,45 \\
-34\end{array}$ & $\begin{array}{l}-1,62 \\
-125,6\end{array}$ & $\begin{array}{l}-0,48 \\
-37,2\end{array}$ & $\begin{array}{l}-1,92 \\
-113,6\end{array}$ & $\begin{array}{l}-0,51 \\
-30,4\end{array}$ & $\begin{array}{l}-2,47 \\
-95,7\end{array}$ & $\begin{array}{l}-0,59 \\
-22,9\end{array}$ & $\begin{array}{l}-10,31-3,04 \\
-104,9-30,9\end{array}$ \\
\hline $\begin{array}{l}\text { Nettobilanz } B_{n} \\
b_{n}=B_{n} / S\end{array}$ & $\begin{array}{l}10^{6} \mathrm{~m}^{3} \text { w.e. } \\
\mathrm{cm} \text { w.e. }\end{array}$ & $\begin{array}{l}-1,71 \\
-40\end{array}$ & $\begin{array}{l}-2,35 \\
-55\end{array}$ & $\begin{array}{l}-0,68 \\
-15,9\end{array}$ & $\begin{array}{l}-1,1 \\
-85,3\end{array}$ & $\begin{array}{l}-0,32 \\
-24,8\end{array}$ & $\begin{array}{l}-1,11 \\
-65,7\end{array}$ & $\begin{array}{l}-0,01 \\
-1,3\end{array}$ & $\begin{array}{l}-1,49 \\
-58,1\end{array}$ & $\begin{array}{l}-0,15 \\
-5,8\end{array}$ & $\begin{array}{l}-6,05-1,16 \\
-61,6-11,8\end{array}$ \\
\hline
\end{tabular}


In der gleichen Zeitspanne wurden hingegen im Sommer 197610 Schneetage festgestellt. Die wiederholten Schneeablagerungen auf der aperen Eisoberfläche vermindern die Ablation. Bevor weitere Eisablation stattfinden kann, muß zuerst die Schneeschicht geschmolzen werden. Daraus resultiert ein verminderter Massenverlust.

Verschiedene Beobachtungen deuten an, daß das Laika Ice Cap schon früher eine Reihe von negativen Haushaltsjahren gehabt haben muß. Auf den schuttbedeckten Hängen seitlich über dem Gletscher lassen sich an gewissen Stellen scharfe Linien feststellen. Diese sogenannte «trim line» ist eine Grenze zwischen dem mit Flechten bewachsenen Geröll und dem nackten, unbewachsenen Hang, der erst später vom Eis befreit und durch Pionierpflanzen besiedelt wurde. Beim Ice Wall Glacier verläuft diese Grenze etwa $20 \mathrm{~m}$ über der heutigen Eisoberfläche; beim Laika Glacier liegt auf der orographisch rechten Seite eine markante «trim line» etwa $30 \mathrm{~m}$ über dem Gletscherrand.

Durch Vergleich von Luftbildern aus den Jahren 1959 und 1971 läßt sich ebenfalls ein langfristiger Volumenverlust des Laika Glacier, wenigstens für das Zungengebiet, erfassen.

Eine photogrammetrische Auswertung dieser Bilder ergab eine Absenkung der Eishöhe vor allem im Gebiet der Verengung des Gletschers oberhalb der Zunge. Der über das Querprofil gemittelte Gletscherschwund betrug für diese 12 Jahre $14,5 \mathrm{~m}$, was einer jährlichen Absenkung von nahezu 1,3 m entspricht. Dementsprechend wurde das Zungengebiet vor 12 Jahren mit mehr Eis aus dem Akkumulationsgebiet versorgt.

\section{Zusammenfassung}

Aus dem Verhalten der Laika-Gletscherzunge läßt sich mit einiger Vorsicht auf die Veränderungen der ganzen Eiskappe schließen. Die während nur zwei Jahren beobachteten übrigen Komponenten der Eiskappe, nämlich die des Wulf und Ice Wall Glacier sowie der Restfläche weisen ähnlich negative Massenbilanzwerte auf. Abschließend läßt sich aufgrund des durchgehend negativen Massenhaushaltes der letzten drei Jahre feststellen, daß der beträchtliche Massenschwund, der durch Luftfotos für die Periode 1959-1971 belegt ist, auch heute noch andauert und daß daher die heutigen Gletscher der südöstlichen Coburg Island immer noch größere Abmessungen haben als dem derzeitigen Klima entspricht. Es wurde festgestellt, daß die Bilanzunterschiede der einzelnen Jahre durch Temperaturdifferenzen und unterschiedliche Niederschlagsverteilung während der Hauptablationsperiode bestimmt werden.

\section{Verdankung}

Die vorliegende Arbeit wurde im Rahmen des «North Water Project», das gemeinsam durch die kanadische Regierung (Department of Environment), die US Science Foundation und den Schweizerischen Nationalfonds finanziert wurde, durchgeführt. Die Mithilfe einer Reihe von Expeditionsteilnehmern im Feld, im besonderen die Vorarbeiten von G.Kappenberger, seien hiermit bestens verdankt.

\section{Literatur}

BAIRD, P. O. 1952. Method of nourishment of the Barnes Ice Cap. Journal of Glaciology, Vol. 2, No. 11, p. 2-9. HOINKES, H. 1970. Methoden und Möglichkeiten von Massenhaushaltsstudien auf Gletschern. Zeitschrift für Gletscherkunde und Glazialmorphologie, Bd.6, Heft 1-2, p. 37-90.

KAPPENBERGER, G. 1974. Massenhaushalt und Bewegung des Laikagletschers, Coburg Island, NWT, 1973/ 1974. Diplomarbeit, ausgeführt am Geographischen Institut ETHZ. 